\title{
Applications of Differential Subordination for Argument Estimates of Multivalent Analytic Functions
}

\author{
Meng-Ting Lu, Ting Jia, Xing-Qian Ling, and Jin-Lin Liu \\ Department of Mathematics, Yangzhou University, Yangzhou 225002, China \\ Correspondence should be addressed to Jin-Lin Liu; jlliu@yzu.edu.cn \\ Received 21 November 2013; Accepted 20 January 2014; Published 26 February 2014 \\ Academic Editor: David Kalaj
}

Copyright (C) 2014 Meng-Ting Lu et al. This is an open access article distributed under the Creative Commons Attribution License, which permits unrestricted use, distribution, and reproduction in any medium, provided the original work is properly cited.

By using the method of differential subordinations, we derive some properties of multivalent analytic functions. All results presented here are sharp.

This paper is dedicated to Professor Miodrag Mateljević on the occasion of his 65th birthday

\section{Introduction}

Let $A(p)$ denote the class of functions $f(z)$ of the form

$$
f(z)=z^{p}+\sum_{n=1}^{\infty} a_{p+n} z^{p+n} \quad(p \in N=\{1,2,3, \ldots\}),
$$

which are analytic in the open unit disk $D=\{z \in C:|z|<1\}$. Let $f(z)$ and $g(z)$ be analytic in $D$. Then, we say that $f(z)$ is subordinate to $g(z)$ in $D$, written as $f(z) \prec g(z)$, if there exists an analytic function $w(z)$ in $D$, such that $|w(z)| \leq|z|$ and $f(z)=g(w(z))(z \in D)$. If $g(z)$ is univalent in $D$, then the subordination $f(z) \prec g(z)$ is equivalent to $f(0)=g(0)$ and $f(D) \subset g(D)$. Let $p(z)=1+p_{1} z+\cdots$ be analytic in $D$. Then, for $-1 \leq B<A \leq 1$, it is clear that

$$
p(z) \prec \frac{1+A z}{1+B z} \quad(z \in D)
$$

if and only if

$$
\begin{gathered}
\left|p(z)-\frac{1-A B}{1-B^{2}}\right|<\frac{A-B}{1-B^{2}} \quad(-1<B<A \leq 1 ; z \in D), \\
\operatorname{Re} p(z)>\frac{1-A}{2} \quad(B=-1 ; z \in D) .
\end{gathered}
$$

Recently, a number of results for argument properties of analytic functions have been obtained by several authors (see, e.g., [1-5]). The objective of the present paper is to derive some further interesting properties of multivalent analytic functions. The basic tool used here is the method of differential subordinations.

To derive our results, we need the following lemmas.

Lemma 1 (see [6, Theorem 1, page 776]). Let $h(z)$ be analytic and starlike univalent in $D$ with $h(0)=0$. If $g(z)$ is analytic in $D$ and $z g^{\prime}(z) \prec h(z)$, then

$$
g(z) \prec g(0)+\int_{0}^{z} \frac{h(t)}{t} d t .
$$

Lemma 2 (see [5, Theorem 1, page 1814]). Let $0<\alpha_{1} \leq 1$, $0<\alpha_{2} \leq 1, \beta=\left(\alpha_{1}-\alpha_{2}\right) /\left(\alpha_{1}+\alpha_{2}\right)$, and $c=e^{\beta \pi i}$. Also let

$$
\lambda_{0} a \geq 0, \quad \lambda(b+2) \geq 0, \quad(b+1) \operatorname{Re} \mu \geq 0,
$$

$$
|b+1| \leq \frac{2}{\alpha_{1}+\alpha_{2}}, \quad|a-b-1| \leq \frac{1}{\max \left\{\alpha_{1}, \alpha_{2}\right\}} .
$$

If $q(z)$ is analytic in D with $q(0)=1$ and

$$
\begin{aligned}
\lambda_{0}(q(z))^{a}+\lambda(q(z))^{b+2} & +\mu(q(z))^{b+1} \\
& +z q^{\prime}(z)(q(z))^{b} \prec h(z) \quad(z \in D),
\end{aligned}
$$


where

$$
\begin{aligned}
h(z)= & \lambda_{0}\left(\frac{1+c z}{1-z}\right)^{a\left(\left(\alpha_{1}+\alpha_{2}\right) / 2\right)}+\left(\frac{1+c z}{1-z}\right)^{(1 / 2)(b+1)\left(\alpha_{1}+\alpha_{2}\right)} \\
& \times\left(\mu+\lambda\left(\frac{1+c z}{1-z}\right)^{\left(\alpha_{1}+\alpha_{2}\right) / 2}\right. \\
& \left.+\frac{\alpha_{1}+\alpha_{2}}{2}\left(\frac{z}{1-z}+\frac{c z}{1+c z}\right)\right)
\end{aligned}
$$

is (close-to-convex) univalent in $D$, then

$$
-\frac{\pi}{2} \alpha_{2}<\arg (q(z))<\frac{\pi}{2} \alpha_{1} \quad(z \in D)
$$

The bounds $\alpha_{1}$ and $\alpha_{2}$ in (9) are sharp for the function $q(z)$ defined by

$$
q(z)=\left(\frac{1+c z}{1-z}\right)^{\left(\alpha_{1}+\alpha_{2}\right) / 2}
$$

Remark 3 (see [5, Lemma 2, page 1813]). The function $q(z)$ defined by (10) is analytic and univalent convex in $D$ and

$$
q(D)=\left\{w: w \in C,-\frac{\pi}{2} \alpha_{2}<\arg w<\frac{\pi}{2} \alpha_{1}\right\} .
$$

\section{Main Results}

Our first result is contained in the following.

Theorem 4. Let $\alpha \in(0,1 / 2]$ and $\beta \in(0,1)$. If $f(z) \in A(p)$ satisfies $f(z) \neq 0(0<|z|<1)$ and

$$
\left|\frac{z^{p}}{f(z)}\left(\frac{z f^{\prime}(z)}{f(z)}-p\right)\right|<\delta \quad(z \in D),
$$

where $\delta$ is the smallest positive root of the equation

$$
\alpha \sin \left(\frac{\pi \beta}{2}\right) x^{2}-x+(1-\alpha) \sin \left(\frac{\pi \beta}{2}\right)=0,
$$

then

$$
\left|\arg \left(\frac{f(z)}{z^{p}}-\alpha\right)\right|<\frac{\pi}{2} \beta \quad(z \in D) .
$$

The bound $\beta$ is sharp for each $\alpha \in(0,1 / 2]$.

Proof. Let

$$
g(x)=\alpha \sin \left(\frac{\pi \beta}{2}\right) x^{2}-x+(1-\alpha) \sin \left(\frac{\pi \beta}{2}\right) .
$$

We can see easily that (13) has two positive roots. Since $g(0)>$ 0 and $g(1)<0$, we have

$$
0<\frac{\alpha}{1-\alpha} \delta \leq \delta<1
$$

Put

$$
\frac{f(z)}{z^{p}}=\alpha+(1-\alpha) p(z)
$$

Then, from the assumption of the theorem, we can see that $p(z)$ is analytic in $D$ with $p(0)=1$ and $\alpha+(1-\alpha) p(z) \neq 0$ for all $z \in D$. Taking the logarithmic differentiations in both sides of (17), we get

$$
\begin{gathered}
\frac{z f^{\prime}(z)}{f(z)}-p=\frac{(1-\alpha) z p^{\prime}(z)}{\alpha+(1-\alpha) p(z)} \\
\frac{z^{p}}{f(z)}\left(\frac{z f^{\prime}(z)}{f(z)}-p\right)=\frac{(1-\alpha) z p^{\prime}(z)}{(\alpha+(1-\alpha) p(z))^{2}}
\end{gathered}
$$

for all $z \in D$. Thus, inequality (12) is equivalent to

$$
\frac{(1-\alpha) z p^{\prime}(z)}{(\alpha+(1-\alpha) p(z))^{2}} \prec \delta z .
$$

By using Lemma 1, (20) leads to

$$
\int_{0}^{z} \frac{(1-\alpha) p^{\prime}(t)}{(\alpha+(1-\alpha) p(t))^{2}} d t \prec \delta z
$$

or to

$$
1-\frac{1}{\alpha+(1-\alpha) p(z)} \prec \delta z .
$$

According to (16), (22) can be written as

$$
p(z) \prec \frac{1+(\alpha /(1-\alpha)) \delta z}{1-\delta z} .
$$

Now, by taking $A=(\alpha /(1-\alpha)) \delta$ and $B=-\delta$ in (2) and (3), we have

$$
\begin{aligned}
\left|\arg \left(\frac{f(z)}{z^{p}}-\alpha\right)\right| & =|\arg p(z)| \\
& <\arcsin \left(\frac{\delta}{1-\alpha+\alpha \delta^{2}}\right)=\frac{\pi}{2} \beta
\end{aligned}
$$

for all $z \in D$ because of $g(\delta)=0$. This proves (14).

Next, we consider the function $f(z)$ defined by

$$
f(z)=\frac{z^{p}}{1-\delta z}
$$

for all $z \in D$. It is easy to see that

$$
\left|\frac{z^{p}}{f(z)}\left(\frac{z f^{\prime}(z)}{f(z)}-p\right)\right|=|\delta z|<\delta
$$

for all $z \in D$. Since

$$
\frac{f(z)}{z^{p}}-\alpha=(1-\alpha) \frac{1+(\alpha /(1-\alpha)) \delta z}{1-\delta z},
$$

it follows from (3) that

$$
\sup _{z \in U}\left|\arg \left(\frac{f(z)}{z^{p}}-\alpha\right)\right|=\arcsin \left(\frac{\delta}{1-\alpha+\alpha \delta^{2}}\right)=\frac{\pi}{2} \beta .
$$

Hence, we conclude that the bound $\beta$ is the best possible for each $\alpha \in(0,1 / 2]$.

Next, we derive the following. 
Theorem 5. If $f(z) \in A(p)$ satisfies $f(z) \neq 0(0<|z|<1)$ and

$$
\operatorname{Re}\left\{\frac{z^{p}}{f(z)}\left(\frac{z f^{\prime}(z)}{f(z)}-p\right)\right\}<\gamma \quad(z \in D)
$$

where

$$
0<\gamma<\frac{1}{2 \log 2}
$$

then

$$
\operatorname{Re} \frac{z^{p}}{f(z)}>1-2 \gamma \log 2 \quad(z \in D) .
$$

The bound in (31) is sharp.

Proof. Let

$$
p(z)=\frac{f(z)}{z^{p}} .
$$

Then, from the assumption of the theorem we can see that $p(z)$ is analytic in $D$ with $p(0)=1$ and $p(z) \neq 0$ for all $z \in D$. According to (32) and (29), we have immediately

$$
1-\frac{z p^{\prime}(z)}{\gamma p^{2}(z)} \prec \frac{1+z}{1-z}
$$

that is,

$$
z\left(\frac{1}{p(z)}\right)^{\prime} \prec \frac{2 \gamma z}{1-z}
$$

Now, by using Lemma 1 , we obtain

$$
\frac{1}{p(z)} \prec 1-2 \gamma \log (1-z) .
$$

Since the function $1-2 \gamma \log (1-z)$ is convex univalent in $D$ and

$$
\operatorname{Re}(1-2 \gamma \log (1-z))>1-2 \gamma \log 2 \quad(z \in D),
$$

from (35), we get inequality (31).

To show that the bound in (31) cannot be increased, we consider

$$
f(z)=\frac{z^{p}}{1-2 \gamma \log (1-z)} \quad(z \in D) .
$$

It is easy to verify that the function $f(z)$ satisfies inequality (29). On the other hand, we have

$$
\operatorname{Re} \frac{z^{p}}{f(z)} \longrightarrow 1-2 \gamma \log 2
$$

as $z \rightarrow-1$. Now, the proof of the theorem is complete.

Finally, we discuss the following theorem.
Theorem 6. Let $\alpha, \gamma \in(0,1)$. If $f(z) \in A(p)$ satisfies $f(z) \neq 0(0<|z|<1)$ and

$$
\begin{gathered}
\mid \arg \left\{\frac{f(z)}{z^{p}}\left(\gamma\left(\frac{z f^{\prime}(z)}{f(z)}-p\right)+(1-\gamma) \frac{f(z)}{z^{p}}\right)\right. \\
\left.-(1-\gamma) \alpha^{2}\right\} \mid<\pi \delta
\end{gathered}
$$

for all $z \in D$, where

$$
\delta=\frac{1}{2}+\frac{1}{\pi} \tan ^{-1}\left(\frac{\sqrt{\gamma(2(1-\alpha)(1-\gamma)+\gamma)}}{2 \alpha(1-\gamma)}\right),
$$

then

$$
\operatorname{Re} \frac{f(z)}{z^{p}}>\alpha \quad(z \in D) .
$$

The bound $\delta$ in (39) is sharp.

Proof. Define the function $p(z)$ by (17). For $\alpha, \gamma \in(0,1)$, it follows from (17) and (18) that

$$
\begin{aligned}
\frac{1}{\gamma(1-\alpha)}\left\{\frac{f(z)}{z^{p}}\left(\gamma\left(\frac{z f^{\prime}(z)}{f(z)}-p\right)+(1-\gamma) \frac{f(z)}{z^{p}}\right)\right. \\
\left.-(1-\gamma) \alpha^{2}\right\} \\
=\frac{(1-\alpha)(1-\gamma)}{\gamma} p^{2}(z)+\frac{2 \alpha(1-\gamma)}{\gamma} p(z)+z p^{\prime}(z)
\end{aligned}
$$

for all $z \in D$. Putting

$$
\begin{gathered}
a=b=\lambda_{0}=0, \quad \alpha_{1}=\alpha_{2}=1, \\
\lambda=\frac{(1-\alpha)(1-\gamma)}{\gamma}, \quad \mu=\frac{2 \alpha(1-\gamma)}{\gamma}
\end{gathered}
$$

in Lemma 2 and using (42), we see that if

$$
\begin{gathered}
\frac{1}{\gamma(1-\alpha)}\left\{\frac{f(z)}{z^{p}}\left(\gamma\left(\frac{z f^{\prime}(z)}{f(z)}-p\right)+(1-\gamma) \frac{f(z)}{z^{p}}\right)\right. \\
\left.-(1-\gamma) \alpha^{2}\right\} \prec h(z),
\end{gathered}
$$

where

$$
\begin{aligned}
& h(z) \\
& \qquad \begin{array}{l}
\left(\frac{1+z}{1-z}\right)\left(\frac{(1-\alpha)(1-\gamma)}{\gamma}\left(\frac{1+z}{1-z}\right)+\frac{2 \alpha(1-\gamma)}{\gamma}\right. \\
\left.+\frac{2 z}{1-z^{2}}\right),
\end{array}
\end{aligned}
$$

then (41) holds true. 
Letting $0<\theta<\pi$ and $x=\cot (\theta / 2)$, we deduce that

$$
\begin{aligned}
& \arg h\left(e^{i \theta}\right) \\
& =\frac{\pi}{2}+\arg \left\{\frac{(1-\alpha)(1-\gamma)}{\gamma} x e^{\pi i / 2}+\frac{2 \alpha(1-\gamma)}{\gamma}\right. \\
& \left.+\frac{i}{2}\left(x+\frac{1}{x}\right)\right\} \\
& =\frac{\pi}{2}+\tan ^{-1}\left(\frac{(2(1-\alpha)(1-\gamma)+\gamma) x^{2}+\gamma}{4 \alpha(1-\gamma) x}\right) .
\end{aligned}
$$

Making use of (46), we obtain that

$$
\begin{aligned}
& \inf _{|z|=1(z \neq \pm 1)}|\arg h(z)| \\
& \quad=\min _{0<\theta<\pi} \arg h\left(e^{i \theta}\right) \\
& =\frac{\pi}{2}+\min _{x>0} \tan ^{-1}\left(\frac{(2(1-\alpha)(1-\gamma)+\gamma) x^{2}+\gamma}{4 \alpha(1-\gamma) x}\right) \\
& =\frac{\pi}{2}+\tan ^{-1}\left(\frac{\sqrt{\gamma(2(1-\alpha)(1-\gamma)+\gamma)}}{2 \alpha(1-\gamma)}\right) \\
& =\pi \delta .
\end{aligned}
$$

Therefore, if $f(z) \in A(p)$ satisfies (39), then the subordination (44) holds, and, thus, we obtain (41).

For the function

$$
\frac{f(z)}{z^{p}}=\frac{1+(1-2 \alpha) z}{1-z}
$$

we find that

$$
\begin{gathered}
\frac{1}{\gamma(1-\alpha)}\left\{\frac{f(z)}{z^{p}}\left(\gamma\left(\frac{z f^{\prime}(z)}{f(z)}-p\right)+(1-\gamma) \frac{f(z)}{z^{p}}\right)\right. \\
\left.-(1-\gamma) \alpha^{2}\right\}=h(z),
\end{gathered}
$$

where $h(z)$ is defined by (45). In view of (46) and (49), we conclude that the bound $\delta$ in (39) is the largest number such that (41) holds true. This completes the proof.

\section{Conflict of Interests}

The authors declare that there is no conflict of interests regarding the publication of this paper.

\section{Acknowledgment}

The authors would like to express their sincere thanks to the referees for careful reading and suggestions which helped them improve the paper.

\section{References}

[1] A. Gangadharan, V. Ravichandran, and T. N. Shanmugam, "Radii of convexity and strong starlikeness for some classes of analytic functions," Journal of Mathematical Analysis and Applications, vol. 211, no. 1, pp. 301-313, 1997.

[2] J.-L. Liu, "The Noor integral and strongly starlike functions," Journal of Mathematical Analysis and Applications, vol. 261, no. 2, pp. 441-447, 2001.

[3] M. Nunokawa, S. Owa, E. Y. Duman, and M. Aydoğan, "Some properties of analytic functions relating to the Miller and Mocanu result," Computers \& Mathematics with Applications, vol. 61, no. 5, pp. 1291-1295, 2011.

[4] N.-E. Xu, D.-G. Yang, and S. Owa, "On strongly starlike multivalent functions of order $\beta$ and type $\alpha$," Mathematische Nachrichten, vol. 283, no. 8, pp. 1207-1218, 2010.

[5] D.-G. Yang and J.-L. Liu, "Argument inequalities for certain analytic functions," Mathematical and Computer Modelling, vol. 52, no. 9-10, pp. 1812-1821, 2010.

[6] T. J. Suffridge, "Some remarks on convex maps of the unit disk," Duke Mathematical Journal, vol. 37, no. 4, pp. 775-777, 1970. 


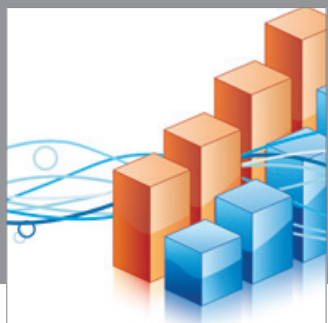

Advances in

Operations Research

mansans

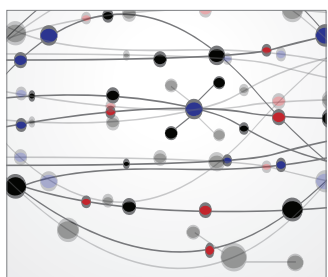

The Scientific World Journal
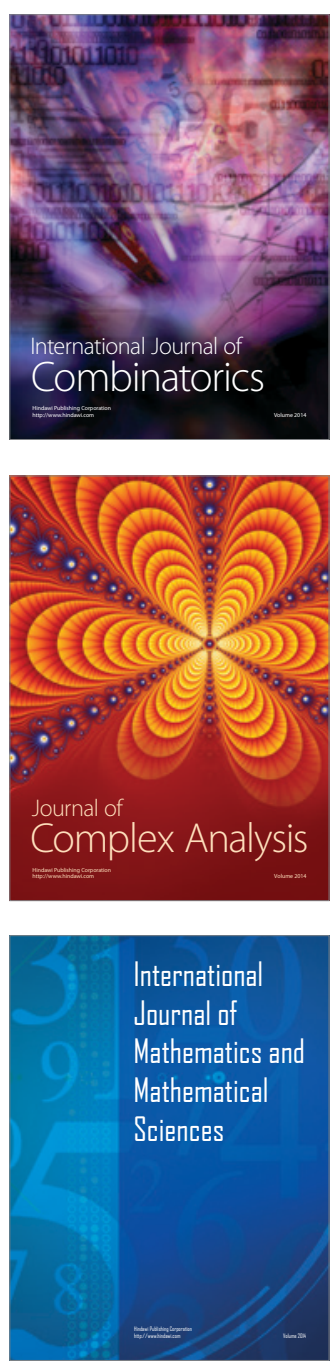
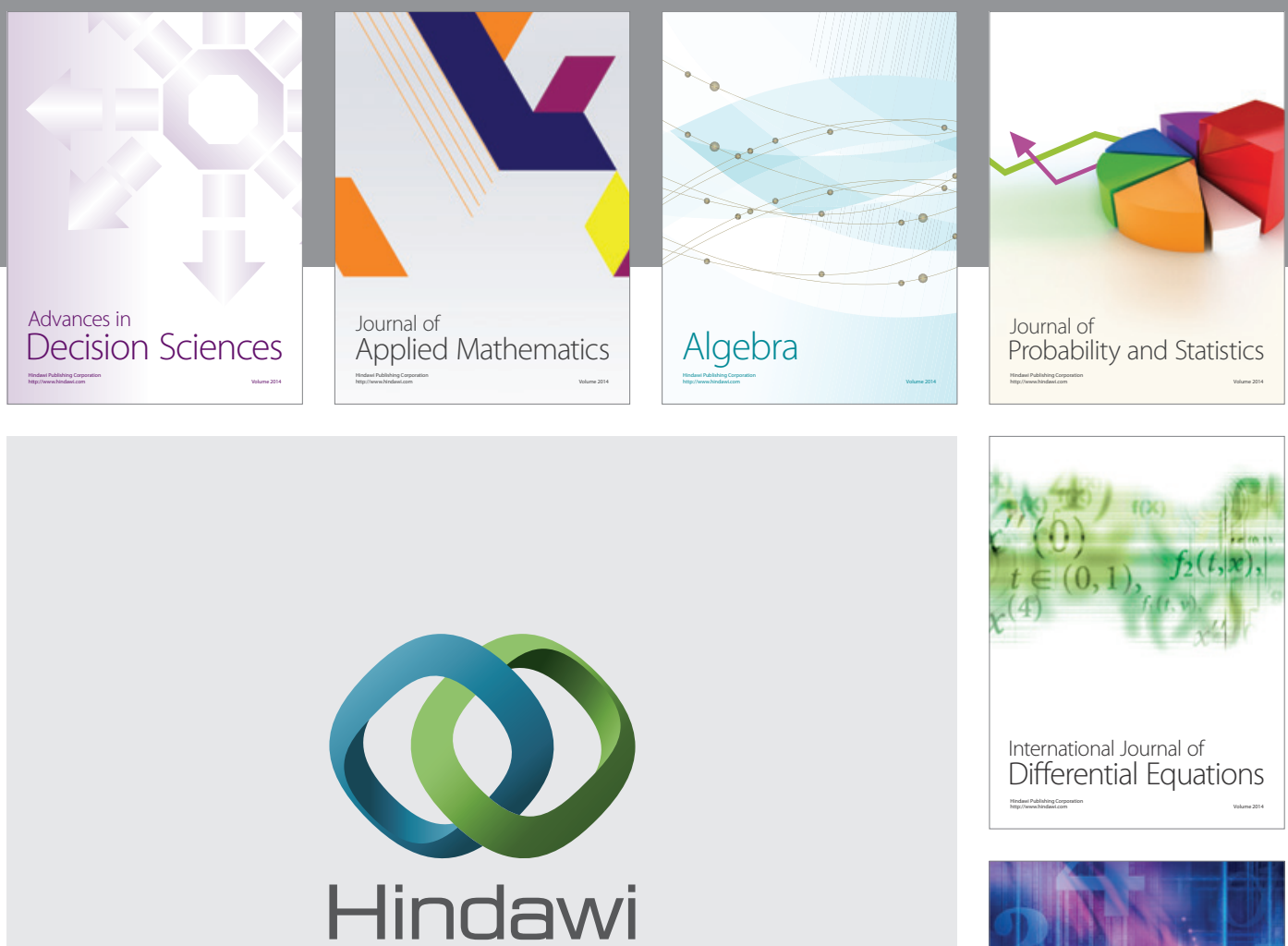

Submit your manuscripts at http://www.hindawi.com
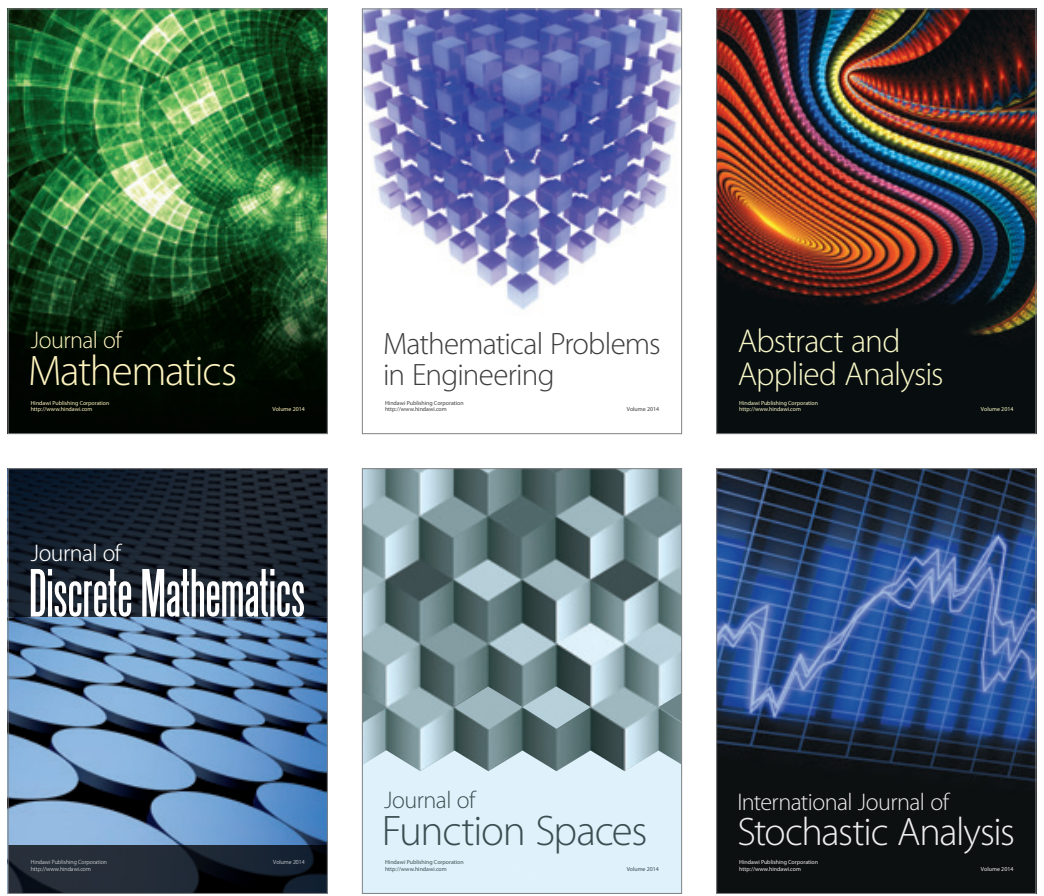

Journal of

Function Spaces

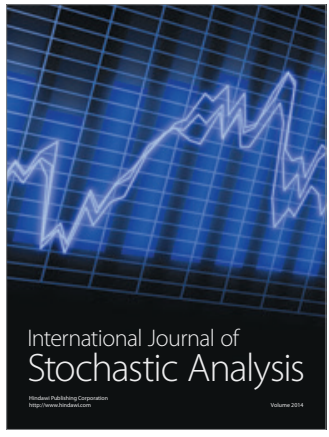

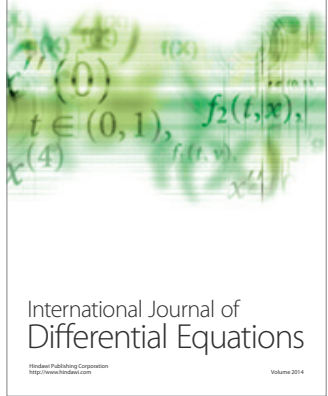
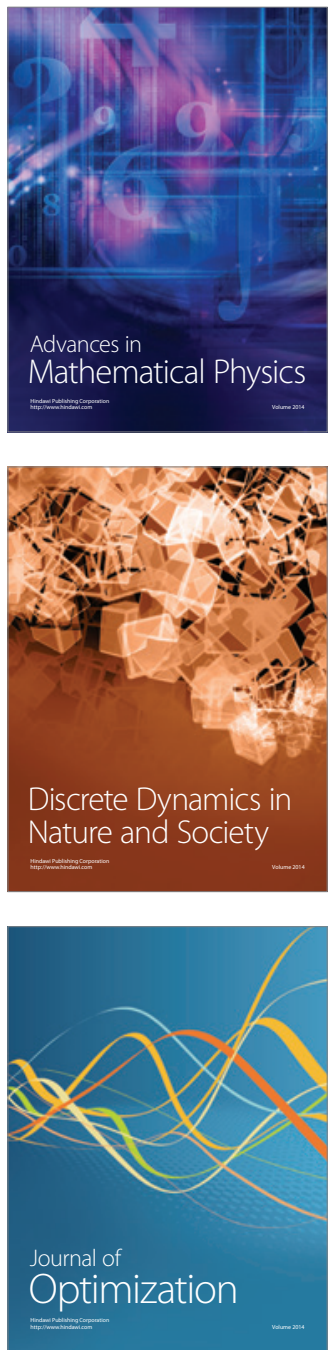